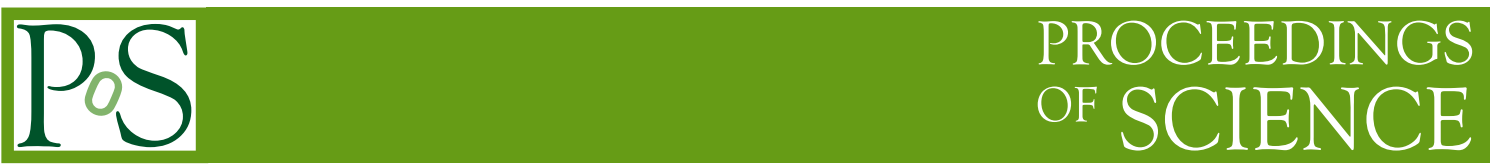

\title{
Aspects of Nonlocal Models of Modified Einsteinian Gravity
}

\author{
Emilio Elizalde* \\ Consejo Superior de Investigaciones Científicas \\ Instituto de Ciencias del Espacio (ICE-CSIC) \\ Institut d'Estudis Espacials de Catalunya (IEEC) \\ Campus UAB, Facultat de Ciències, Torre C5-Parell-2a planta \\ 08193 Bellaterra (Barcelona) Spain \\ E-mail: elizaldedieec.uab.es
}

\begin{abstract}
After a historical introduction, we discuss about independent evidences which have been gathered of the fact that the Universe expansion is accelerating. We then consider some common problems of the postulated models to explain dark energy in a natural way. Afterwards, a family of nonlocal models which exhibits a number of nice properties is introduced. Basic mathematical issues in relation with this model and some very promising initial results obtained in our study, together with several problems we still have to face, and other interesting questions for further work, are discussed.
\end{abstract}

$3 d$ International Satellite Conference on Mathematical Methods in Physics - ICMP 2013

21 - 26 October, 2013

Londrina - PR (Brazil)

\footnotetext{
*Speaker.
} 


\section{Introduction: basic cosmological landmarks}

This material corresponds to my lecture, delivered at the Conference on "Mathematical Methods in Physics" held in Londrina (Brazil), in October 2013. In this introduction I will summarize some cosmological developments of the last Century, which may serve as a leading guide to important recent discoveries in cosmology, and to their understanding from fundamental principles, as it already happened in the past in other similar situations. Until the third decade of last Century the Milky Way was believed to be the entire Universe. No wonder since measuring distances has always been, and is still, one of the most difficult—and most important-problems in cosmology. Also, of course, calculating the speeds with which celestial bodies wander about in the sky, be they asteroids, planets or satellites, stars, galaxies or immense clusters, which nevertheless appear as tiny points (or even are not to see at all). During the first twenty of last Century, important discussions among astronomers involved nebulae, in special, spiral nebulae. Tens of thousands of them had been observed already and astronomers thought all these objects were confined to the domains of our own world, namely the Milky Way. Actually, the first nebulae had been already identified nineteen centuries ago by Ptolemy, then by Persian, Arabic and Chinese astronomers, and by Edmond Halley (1715), Charles Messier (1781), and William Herschel and his sister Caroline (1786 and 1802), to mention only a few relevant names, the last having compiled full catalogs of nebuale. On the other hand, the great philosopher Immanuel Kant had advanced the possibility that we lived in an "Island Universe" (as he named it, that is, a universe made of 'islands', ours being just one among many).

On April 26th, 1920, at the Smithsonian Museum of Natural History in Washington DC, a debate took place (termed now the Great Debate, [四]), between two important astronomers, Harlow Shapley and Heber Curtis, on "The Scale of the Universe". Shapley defended that the Milky Way was the whole Universe and Curtis countered that Andromeda, and possibly other nebulae, were in fact "island universes" by themselves. Curtis had found, in proportion, many more novae in Andromeda than in the rest of the Milky Way and argued that it would be very unlikely to have so many novae concentrated in one small section, to what he added that the novae in Andromeda were fainter than those in other regions of the Milky Way, what was also a proof that Andromeda was outside of it (some 150,000 Mpc away from us, he calculated). Curtis also mentioned the Doppler redshifts of many nebulae, which could be interpreted by saying that they were going away from us. Although these arguments seem very convincing now, they were not quantitatively conclusive and, in the end, none of the two contenders won the day. However, the suspicion had been created among the open minded that many of the nebulae were probably other galaxies, and several attempts at calculating the distance to Andromeda appeared in the following years.

Just four years later Edwin Hubble was able to prove that Andromeda was beyond the limits of our own galaxy. He could identify Cepheid variable stars in several spiral nebulae, including Andromeda and the Triangulum, and thus make use, to calculate the distances, of Henrietta S. Leavitt's period-luminosity relationship, which she had obtained in 1912 (after years of tough analysis of thousands of these stars): a straight line connecting the luminosity and the logarithm of the period of variability of Cepheids [3]. Hubble published his finding in the New York Times, on November 23, 1924 []], and presented them later at the January 1, 1925, meeting of the American Astronomical Society. Again a few years later he derived his celebrated law: velocity-distance re- 
lationship for nebulae, containing his famous constant. He used this time, as he duely recognized ${ }^{1}$, the long and accurate table of velocities of nebulae that Vesto Slipher had compiled, starting in 1912 with the first four redshifts, at the Lowell Observatory in Arizona, in the form of redshifts of their spectral lines.

\section{Mathematical approaches}

Turning now to more mathematical approaches, we should recall that when Albert Einstein constructed-in 1915 and starting from very basic physical postulates, such as the principles of covariance and equivalence of the laws of physics—his theory of General Relativity (GR), scientists (himself included) where firmly convinced that our universe was static-what does not mean that celestial bodies did not move, but that stars and their clusters, in their wandering and distribution, would always have remained as we see them at present, with no essential changes. To his disappointment, he realized that such a universe was not compatible with his field equations: a static universe could not be stable, since it would always collapse due to the attraction of gravity. But he came up with a solution, since he had the remaining freedom to introduce in his equations a constant multiplied by the metric tensor (the now very famous cosmological constant). The problem was still that it had no physical interpretation but, endowed with the right sign, it did produce a repulsive pressure to counter the gravitational attraction and keep the universe solution static.

Already in 1915 Karl Schwarzschild found a solution to Einstein's equations, which corresponds to what is now known as a black hole (his insight was to look for the simplest, spherically symmetrical solution). And in 1922, Alexander Friedmann obtained another one, by solving the (now called) Friedmann equations, which is even more interesting for cosmology than Schwarzschild's one, because it corresponds to the entire universe. Friedmann's early death prevented him from understanding that his solution actually described an expanding universe. This honor was reserved for Georges Lemaitre who rediscovered essentially the same solution while he was working at the Massachusetts Institute of Technology on his (second) Ph.D. thesis, which he submitted in 1925. Around the same time, Willem de Sitter had also obtained a universe solution (now called de Sitter space), which is the maximally symmetric vacuum solution of Einstein's field equations with a positive (therefore repulsive) cosmological constant. This undoubtedly corresponded to an expanding universe (in fact, in 1917 De Sitter had already theorized that the Universe might be expanding). The problem was that it described a universe devoid of matter, just a vacuum, and thus did not seem to be physically relevant. Nowadays this solution is extremely important as an asymptotic case to describe with good approximation the probable final stage of the evolution of our universe and also its initial stage, the inflationary epoch: the fact that the de Sitter expansion is exactly exponential is extremely helpful in the construction of inflationary models.

\section{Mathematics meets physics}

And now comes the connection. In 1924-25, while working at MIT, Lemaitre visited both Slipher and Hubble to learn about their results. He also attended the meeting of the AAS, in 1925,

\footnotetext{
${ }^{1}$ Hubble acknowledged Slipher's seminal contribution to his own work by declaring that "the first steps in a new field are the most difficult and the most significant. Once the barrier is forced, further development is relatively simple."
} 
where Hubble announced his discovery that certain spiral nebulae were actually separate galaxies and, soon after the meeting, he started to work, for his Thesis, on his own cosmological model. He realized the uniformity of the recession speed of the nebulae, in different directions, and the fact that the redshift seemed to be proportional to the known distances to them, and concluded that this could be better understood not as proper displacements of the galaxies, but much more naturally as a stretching of space itself, an expansion of the cosmos. And this was supported by the fact that his solution to Einstein's equations (recall, the same as Friedmann's) could just be interpreted as corresponding to an expanding Universe. Theory and observations matched! Lemaitre even estimated a rate of expansion close to the figure that Hubble eventually calculated and published two years later. And in 1927, at a meeting in Brussels, he tried to convince Einstein that his static model for the Universe, with a cosmological constant, did not stand, since it was not stable and that, much more important, the cosmological constant was not needed at all. But Einstein was not convinced, quite on the contrary, he dismissed Lemaitre saying that his physical insight was abominable. It took the great genius over two years to understand that, according to his equations, the Universe was indeed expanding and that Lemaitre was absolutely right. Then he abhorred of the cosmological constant by pronouncing his famous sentence: "Away with the cosmological constant. This was the biggest blunder in my life" (in German: "Weg mit der kosmologischen Konstante. Dass war die grösste Eselei meines Lebens"). He realized that, had he truly believed in his field equations, he would have predicted that the Universe was actually expanding (and not static), even before astronomers obtained their data on redshifts ${ }^{2}$. In 1965 the expansion of our Universe was undoubtedly detected by Arno A. Penzias and Robert W. Wilson [田], at the Bell Laboratories in New Jersey. They got the 1978 Nobel Prize in Physics for their very important observation, which can be considered as one the most important ever made in Human History. The final conclusions were that the Universe had indeed an origin, a Big Bang, the fabric of space was stretching and, as clearly understood by Lemaitre, Friedmann's solution to Einstein's equations provided a unique (this had been proven by Robertson and Walker in the 1930s), real description of our Universe.

\section{On the acceleration of the Universe expansion}

Until very recently, cosmologists were convinced that the expansion of the Universe, originated in the Big Bang, was uniform, and the main challenge of cosmology was to determine if $\rho$, the mass-energy density of our cosmos was large enough (above critical) so that it would be able to completely stop this expansion at some point in the future ${ }^{3}$ or if, on the contrary, this energy density $\rho$ was smaller, less tan critical and unable to completely stop the Universe expansion. In this case expansion would continue forever, but there was no doubt that the action of gravity would certainly decelerate the expansion rate. The most precise observations then indicated that the value of $\rho$ was seemingly very close to the critical one, $\rho_{c}$, being difficult to determine if it actually was above or below this value. The situation radically changed because of two different analysis of very

\footnotetext{
${ }^{2}$ In quite the same way as Dirac predicted later the existence of the positive electron, the positron, because it was a second solution of his quantum equation for the electron, impossible to get rid off by reasonable arguments.

${ }^{3} \mathrm{An}$ instant from which the Universe would stop expanding and begin to contract, to finally finish in a so-called Big Crunch.
} 
precise observations carried out with the big Hubble Space Telescope on type Ia supernovae by two teams. Both wanted to measure with high precision the deceleration, caused by gravity attraction, on the expansion rate of the Universe, by accurately calibrating the variation in the expansion rate with distance. To their enormous surprise, the results obtained were completely unexpected, and matched with each other. The High-z Supernova Search Team an Australian-American project, led by Brian Schmidt and Adam Riess published first results in 1998 []], an the Supernova Cosmology Project led by Saul Perlmutter at Lawrence Berkeley National Laboratory, published independent results in 1999 [目]. The common conclusion was that the expansion of the Universe is nowadays accelerating, and not decelerating, and that it has been accelerating for a long period of time in the past. The impact of this discovery on our knowledge of the Universe was so extraordinary that the three mentioned researchers were awarded the 2011 Nobel Prize in Physics. The consequences of this discovery are enormous since, in order that this acceleration can stand a force must be present, a force acting constantly at the level of the whole cosmos. The question is, what kind of force can have this property in order to produce the desired acceleration?

At the beginning it was not so clear that the only interpretation for the Type Ia Supernovae results could be that the Universe expansion was accelerating. This was controverted for some time and other possible explanations - as those based on a privileged position of our local galaxy group in the cosmos-were published. We could be in one of the enormous voids surrounded by very massive structures, well known from large scale structure plots since the very first map of a slice of the Universe was obtained [ $\left[\mathbb{}\right.$ ], by de Lapparent, Geller and Huchra in $1986^{4}$, and other alternative interpretations appeared, too, as possible additional influence of dust in the measurements. However, Type Ia Supernovae have been confirmed to be very good standard candles for the observed redshift range. Indeed, they exhibit a strong and consistent brightness along considerable cosmological distances. And, what is more important, since 1990 many other independent proofs have been developed which can confirm the results, as the impact of the Universe acceleration on the fluctuations of the cosmic microwave background (CMB). Thus, the imprint of the acceleration on the gravitational potential wells which contribute to the integrated Sachs-Wolfe effect has been obtained, and then translated into colder and hotter spots in the CMB map. Also, the effect of the acceleration on gravitational lensing, and on the large scale structure of the Universe, as a correction to the baryon acoustic oscillations (BAO), which were produced at the instant of decoupling of radiation from matter. These observations are very much independent from each other and there may be little doubt left today that the Universe expansion is accelerating, and that this has been the case during a long period of the Universe history. The Dark Energy Survey project (DES) will provide new measurements, and this is a serious comprehensive mission which will integrate all those very different techniques.

To summarize what has been said above, three main kind of approaches have been devised in order to measure the effects of dark energy on the expansion rate of our Universe, namely

1. Standard candles, in particular Type Ia Supernovae, but also other objects are envisaged for the near future; here one will measure the luminosity distance as a function of the redshift.

\footnotetext{
${ }^{4}$ A milestone of the CfA Redshift Survey started in 1977 by Marc Davis, John Huchra, Dave Latham and John Tonry
} 
2. Standard rulers, as is the case of the aforementioned BAO oscillations, which will allow to measure angular diametral distances and the expansion rate, both as a function of the redshift.

3. The growth of the fluctuations generated at the origin of the Universe, which were immediately amplified by inflation, and even maybe fluctuations in the process of recombination too.

4. Angular diameters and luminosity distances are always integrals of the inverse expansion rate of the Universe and, in this way, they encode the effects of dark energy.

It must be remarked that in order to get competitive constraints on the dark energy we will have to detect changes in the Hubble constant as a function of the redshift, $H(z)$, at the order of one percent. This is predicted to give statistical errors in the dark energy equation of state (EoS) of the order of ten percent, what will give some possibility to discriminate among the many different models that are already in the market.

To this end it will be necessary

1. To calibrate the above mentioned rulers to great accuracy over a good part of the age of our Universe.

2. To measure the rulers over much of the total volume of our Universe.

3. And to make, in any case, very precise measurements of the rulers, in order to be able to be predictive and discriminative.

4. When dealing with large scales or in early times, the perturbative treatment is considered to be valid (at least in the more standard theories) and calculations are expected to be under control.

To repeat, length scales from physics of the early universe are imprinted on distribution of mass and radiation that we observe, since they form indeed time-independent rulers (see a nice description in, e.g., M. White, [[]]). The evidence for the acceleration of the Universe expansion has been presently accumulate from very different, independent observations, as distant supernovae, the cosmic microwave background, baryon acoustic oscillations, the galaxy distribution, and correlations of cluster distributions and the web structure. All these are indeed multiple and very different sets of evidence which allow to eliminate (or at least highly reduce) possible systematic errors, so that one can reach the conclusion that the expansion of the Universe is accelerating.

Among the most recent projects involved in this observational revolution that we have witnessed during the last couple of decades, in the areas of Astronomy and Cosmology, with an enormous impact on our understanding of the universe, we must mention the projects that first revolutionized our knowledge of the cosmos through detailed observations of the cosmic microwave background (with COBE, several balloons, as BOOMERanG, MAXIMA, HERO, and BLAST, and very specially the Wilkinson Microwave Anisotropy Probe, WMAP), of the large-scale structure of the universe (the Two-Degree-Field, Galaxy Redshift, and Sloan Digital Sky Surveys), and distant supernovae (the Supernovae Projects and other). The use of a variety of instruments of ever increasing precision (on top of the already mentioned, the Hubble Space Telescope, VLT, etc.) 
and the creation of worldwide collaborative consortia (SSDSII, EUCLID, and many other), have opened exciting new prospects for the forthcoming adventure of understanding our Universe. The PLANCK satellite collaboration has started to publish the first cosmological results. A number of ground-based experiments measuring polarization of the CMB (as ACTpol, SPTpol, Polarbear, BICEP, BLISS, etc.) will soon report results, as also the next-generation galaxy surveys (BOSS, DES, HSC, HETDEX, etc.). These new data will allow us to address deep questions, as, e.g., on the nature of dark energy and dark matter, on what did power the Big Bang, on the reality and specific properties of inflation, and on when and how did the first stars and galaxies actually form.

In interpreting these results and trying to understand them from fundamental theories with an important mathematical content, as we will see in what follows, we must keep in mind the lessons we have learned from previous discoveries like the ones discussed above. In the next section, we will be much more specific about the different types of models which are at our disposal in order to explain the acceleration of the Universe expansion, to later concentrate in a specific model we have been working on recently, in further detail.

\section{Alternative models}

In order to try to explain from a mathematical, more or less fundamental model, the present day acceleration of the cosmos two different alternatives appear:

1. We may try to keep General Relativity (GR) at any price, given the enormous successes of this theory during the last and the present Century. As we already remarked, gravity leads to deceleration but in GR pressure also influences geometry, as first clearly discussed by Richard Tolman in 1932 [团], so that negative pressure can drive acceleration. In modern Quantum Field Theory this could be a convenient negative pressure field. Such very far reaching discovery is key to inflationary theories and I have heard Allan Guth refer to it in MIT seminars as the "miracle of Physics \# 1." [ए0]]. The observational evidence would here be explained by an undiscovered substance (field) with negative pressure, the so-called dark energy [ए]].

2. A completely different possibility is to say that GR is wrong, or, in better words, not accurate enough at large scales [12]. In fact, GR was developed using information and intuition at the Solar System scale and it is mainly checked there, although there is also a slowly growing evidence of its validity outside this domain. It is not venturous to guess that GR will need to be modified on large scales, as well as on shorter scales, and the two modifications could even be related ${ }^{5}$. Indeed, some years ago Alexei Starobinsky arrived at this same conclusion, through very different arguments based on the expected quantum corrections to ordinary GR which must occur at very low scales (high energies), which lead to terms of second order in $R$, and higher.

\footnotetext{
${ }^{5}$ This is the aim of the so-called unified models of modified gravity.
} 


\section{Some common problems}

Several important problems appear when one tries to find alternatives to GR. The first one is that we do not have simple guidelines, as gedanken experiment or the like, nor reasons of elegance and simplicity, which were the leading inputs for developing fundamental theories in the past as, e.g., those of Einstein in constructing his theory of gravitation.

Besides that, even if beauty or elegance are abandoned, a modification of gravity will still encounter additional problems (see, e.g., Park and Dodelson [[13]]). Namely,

1. Almost all models contain a mass scale to be set much smaller than any mass found in nature, namely $<10^{-33} \mathrm{eV}$. But then,

(a) what is the possible meaning of this small mass scale?

(b) How can it be protected from interactions with the rest of physics?

2. A severe fine tuning problem in time usually occurs: the modifications to gravity happen to be important right now, and not at previous epochs further in the past.

3. Another problem is the following: modified gravity models must necessarily comply with the celebrated successes of GR in the Solar System domain.

These constraints already discarded ten years ago the very first and most promising modified gravity models which were introduced to explain acceleration [W] and nowadays they still set very tight constraints on many of the new models that appear in the literature, which must eventually be termed as non-viable.

\section{Properties of models with non-local interactions}

A very promising class of modified gravity models which seems to be able to overcome most of these above mentioned problems is due to S. Deser and R. Woodard and contains non-local interactions [44]. To be more specific, these authors consider terms that are functionals of $\square^{-1} R$, with $\square$ the d'Alembertian and $R$ the Ricci scalar, as usual. Good properties of these models are:

1. At cosmological scales the term $\square^{-1} R$ grows slowly:

(a) as $(t / t e q)^{1 / 2}$ in the radiation dominated era, and

(b) logarithmically in the matter dominated era.

2. Thus, at the time of Nucleosynthesis $\square^{-1} R$ is just of order $10^{-6}$.

3. At matter-radiation equilibrium it is only of order one.

4. In a natural way, these terms are thus not relevant at early times and start to influence the dynamics of the Universe only after the matter-radiation transition. This property solves some of the worst fine tuning problems we have mentioned.

5. Since $\square^{-1} R$ is dimensionless, the functional on this term times $R$ has no new mass parameter, what is again very good for the reasons above. 
6. Finally, because $\square^{-1} R$ is extremely small at the Solar System scale, all models of this kind easily pass the local tests of gravity, and are therefore viable.

On top of that, there is some good theoretical motivation for such class of models. Indeed, in relation with this point we can mention the following:

1. In string theory, $R \square^{-1} R$ is precisely the term generated by quantum corrections (away from the critical dimension) as already pointed out by Polyakov [155].

2. It may be possible [ए]] to rewrite these models in terms of local ones with one or more auxiliary scalar fields.

3. Also, non-local interactions could as well describe the early epoch of inflation as has been mentioned above already [ㅁ] ], to wit, a realistic model for the cosmic acceleration, with an arbitrary function of $R \square^{-1} R$, which reproduces the expansion history of $\Lambda \mathrm{CDM}$ can be found in [ए8]. Moreover, a model of this same sort has been used in [ए3] to discuss structure formation in a non-locally modified gravity model.

Before we introduce our own model, some final considerations are still in order.

1. We should note yet that, in spite of all the good properties above, the very first problem we have mentioned still persists: in all these models a completely arbitrary function can be chosen to fit the expansion history.

2. However, let us remark that a very interesting aspect of modified gravity models in general is that, even if they are constructed to reproduce the expansion history equivalent to that of a given dark energy model (such as $\Lambda \mathrm{CDM}$ ), perturbations will often evolve differently than in a model with standard GR plus dark energy, and this will render the modified theories predictive and also falsifiable.

3. Indeed, one way to distinguish DE models from modified gravity is to measure the growth of structure in the Universe. The deviations from dark energy models are expected to be at the 10 to $30 \%$ level and have a truly characteristic signature as a function of the redshift, which suggests that this class of models could be tested by upcoming surveys.

\section{A specific model of type $f\left(\square^{-1} R\right)$}

Let us consider a nonlocal gravity which contains a function of the inverse d'Alembertian, $\square^{-1}$, operator and thus not assuming the existence of a new dimensional parameter in the action. We will focus on the study of cosmological solutions both in the Jordan and in the Einstein frames, including matter in the last case, what is new with respect to previous analysis. We recall that, observationally, the dark energy EoS parameter is now very close to -1, with a tendency to stay below this value, what is somehow intriguing. This number, if finally true, would lead us back to GR with a cosmological constant. But, as of now, a small deviation cannot be excluded by the most accurate astronomical data. Actually, there is some tension between Supernovae and BAO data: while the first favor a value just above -1 , the last ones seem to hint to a value of the 
EoS parameter strictly less than -1 . Anyway, taking into account all available data, neither the sign nor the tendency (e.g., the derivative) of this deviation can be determined at present. This makes room for a number of theoretical models, derived from quite different fundamental theories, which can accommodate such situation. Indeed, several rather simple models are able to reproduce observations, as e.g. the class of so-called quintom models, which involve two fields: a phantom one and an ordinary scalar [ए9].

The nonlocal model we will consider has been usually studied in the Jordan frame, but recently its behavior in the Einstein frame, for the case without matter, was explored too [20]].

We will focus our effort here on the study of:

- cosmological solutions of the model, both in the Jordan and in the Einstein frames;

- explicitly including the case with matter for the last one;

- and we shall consider gravity models with a cosmological constant $\Lambda$ and involving a perfect fluid, to study in detail their cosmological solutions with a power-law cosmic scalar factor: $a \propto t^{n}$. The solutions thus obtained will be proven to generalize other solutions found previously.

Anticipating some result of our study, in the Jordan frame we have obtained an exhaustive class of power-law solutions (we prove that other power-law solutions cannot exist). And we have analyzed in much detail the correspondence between solutions got in the different frames, and proven explicitly how knowledge of power-law solutions in the Jordan's frame can be systematically used to get power-law solutions in the Einstein's one, what will be a generic and quite useful byproduct of our analysis (see also [2] $]$ ).

\subsection{The action}

The action corresponding to our class of nonlocal gravities has the form:

$$
S=\int d^{4} x \sqrt{-g}\left\{\frac{1}{2 \kappa^{2}}\left[R\left(1+f\left(\square^{-1} R\right)\right)-2 \Lambda\right]+\mathscr{L}_{\mathrm{m}}\right\},
$$

where $\kappa^{2}=8 \pi G=8 \pi / M_{\mathrm{Pl}}^{2}, M_{\mathrm{Pl}}=G^{-1 / 2}=1.2 \times 10^{19} \mathrm{GeV}$ is the Planck mass, $f$ a differentiable function (which characterizes the nature of nonlocality), $\square^{-1}$ the inverse of the d'Alembertian operator, $\Lambda$ the cosmological constant, and $\mathscr{L}_{\mathrm{m}}$ the matter Lagrangian. For definiteness, we assume that matter is a perfect fluid. We use the signature $(-,+,+,+)$, with $g$ the determinant of the metric $g_{\mu v}$.

We introduce two scalar fields, namely $\psi=\square^{-1} R$ and a Lagrange multiplier $\xi$

$$
S_{l o c}=\int d^{4} x \sqrt{-g}\left\{\frac{1}{2 \kappa^{2}}[R(1+f(\psi))+\xi(R-\square \psi)-2 \Lambda]+\mathscr{L}_{\mathrm{m}}\right\},
$$

and thus the original non-local action is recast as a local action in the Jordan frame. Varying this action with respect to $\xi$ and $\psi$, one respectively gets the field equations

$$
\square \psi=R, \quad \square \xi=f_{, \psi}(\psi) R,
$$


where $f_{, \psi}(\psi) \equiv \mathrm{d} f / \mathrm{d} \psi$.

The corresponding Einstein equations are obtained by variation of the local action with respect to the metric tensor

$\frac{g_{\mu v}}{2}\left[R \Psi+\partial_{\rho} \xi \partial^{\rho} \psi-2(\Lambda+\square \Psi)\right]-R_{\mu \nu} \Psi-\frac{1}{2}\left(\partial_{\mu} \xi \partial_{\nu} \psi+\partial_{\mu} \psi \partial_{\nu} \xi\right)+\nabla_{\mu} \partial_{\nu} \Psi=-\kappa^{2} T_{(\mathrm{m}) \mu \nu}$,

where $\Psi \equiv 1+f(\psi)+\xi$, and $T_{(\mathrm{m}) \mu \nu}$ is the energy-momentum tensor of the matter sector, given by

$$
T_{(\mathrm{m}) \mu v} \equiv-\frac{2}{\sqrt{-g}} \frac{\delta\left(\sqrt{-g} \mathscr{L}_{\mathrm{m}}\right)}{\delta g^{\mu v}} .
$$

Note that the system of equations here does not include the function $\psi$ itself, but instead $f(\psi)$ and $f_{, \psi}(\psi)$, together with time derivatives of $\psi$. Also, $f(\psi)$ can only be determined up to a constant: one may add a constant to $f(\psi)$ and subtract the same constant from $\xi$ without changing the equations. We shall assume a spatially flat FLRW universe

$$
d s^{2}=-d t^{2}+a^{2}(t) \delta_{i j} d x^{i} d x^{j}
$$

and consider the case where the scalar fields $\psi(t)$ and $\xi(t)$ are functions of the cosmological time tonly.

I this way, the system of equations reduces to

$$
\begin{aligned}
3 H^{2} \Psi & =-\frac{1}{2} \dot{\xi} \dot{\psi}-3 H \dot{\Psi}+\Lambda+\kappa^{2} \rho_{\mathrm{m}}, \\
\left(2 \dot{H}+3 H^{2}\right) \Psi & =\frac{1}{2} \dot{\xi} \dot{\psi}-\ddot{\Psi}-2 H \dot{\Psi}+\Lambda-\kappa^{2} P_{\mathrm{m}}, \\
\ddot{\psi} & =-3 H \dot{\psi}-6\left(\dot{H}+2 H^{2}\right), \\
\ddot{\xi} & =-3 H \dot{\xi}-6\left(\dot{H}+2 H^{2}\right) f_{, \psi}(\psi),
\end{aligned}
$$

where the dot means differentiation with respect to time $t$, in the Jordan frame: $\dot{A}(t) \equiv \mathrm{d} A(t) / \mathrm{d} t$, $H=\dot{a} / a$ being the Hubble parameter. For a perfect matter fluid, $T_{(\mathrm{m}) 00}=\rho_{\mathrm{m}}$ and $T_{(\mathrm{m}) i j}=P_{\mathrm{m}} g_{i j}$. The continuity equation is

$$
\dot{\rho}_{\mathrm{m}}=-3 H\left(P_{\mathrm{m}}+\rho_{\mathrm{m}}\right) .
$$

And by adding these equations we obtain a 2 nd order linear differential equation for $\Psi$, namely,

$$
\ddot{\Psi}+5 H \dot{\Psi}+\left(2 \dot{H}+6 H^{2}\right) \Psi-2 \Lambda+\kappa^{2}\left(P_{\mathrm{m}}-\rho_{\mathrm{m}}\right)=0 .
$$

\subsection{Power-law solutions with $f(\psi)$ a simple exponential function}

Consider the case when $f(\psi)$ is an exponential function

$$
f(\psi)=f_{0} e^{\alpha \psi}
$$

$f_{0}$ and $\alpha$ being nonzero real parameters. The motivations to consider this type of functions are: (i) this is the simplest model available with power-law and de Sitter solutions (only an exponential or a sum of exponentials are possible); (ii) these solutions constitute the better studied case among all possible functions for an expanding universe (with Hubble parameter $H=n / t$, being $n$ a nonzero constant). 
We shall consider matter with the EoS parameter $w_{\mathrm{m}} \equiv P_{\mathrm{m}} / \rho_{\mathrm{m}}$ being a constant but not equal to -1 . For power-law solutions $H=n / t$, the continuity equation has the following general solution ( $\rho_{0}$ is an arbitrary const)

$$
\rho_{\mathrm{m}}(t)=\rho_{0} t^{-3 n\left(w_{\mathrm{m}}+1\right)}
$$

The solutions with $H=n / t$ read

$$
\psi(t)=\psi_{1} t^{1-3 n}-\frac{6 n(2 n-1)}{3 n-1} \ln \left(\frac{t}{t_{0}}\right)
$$

$\psi_{1}, t_{0}$ integration constants. We consider real solutions at $t>0$, hence, $t_{0}>0$. Note these are valid provided $n \neq 1 / 3$ and $n \neq 1 / 2$ (those are special cases, to be specifically considered). There are no power-law solutions in the case $\psi_{1} \neq 0$.

\subsection{Summary of the solutions in the Jordan frame}

For the sake of brevity of our presentation we are only able to provide a summary of the main results obtained after working with this model, namely:

1. Solutions in the Jordan frame for $m \neq 1-3 n$, for $\Lambda=0$ and for $\Lambda \neq 0$.

2. Solutions in the Jordan frame for $m=1-3 n$, for $\Lambda=0$ and for $\Lambda \neq 0$.

3. Local constraints, to wit,

(a) the Newtonian limit of the theory is described by the corresponding local action. One must check whether the power-law solutions found can satisfy this constraint;

(b) depending on the integration constant $\xi_{1}$ being vanishing or not, we can draw very different constraints on the Post-Newtonian parameter $\gamma$ :

i. $\xi_{1} \neq 0$, the constraint can be easily satisfied for a wide range of choices of the parameter $\alpha$;

ii. $\xi_{1}=0$, one needs to tune the parameter $\alpha$ to at least $10^{-5}$ order, to satisfy the local constraint.

Note that, in the previous papers already mentioned, the authors had taken $\xi_{1}=0$ for simplicity. Analysis of the local constraint shows that solutions with nonzero $\xi_{1}$ allow to change the restrictions on the parameter $\alpha$, which are indeed necessary in order to make the model compatible with astronomical observations

4. Power-law solutions for the original nonlocal model.

(a) By varying the nonlocal action with respect to the metric $g_{\mu \nu}$, under the spatially flat FLRW metric, the independent components of the field equations are

$$
3 H^{2}+\Delta G_{00}=\kappa^{2} \rho_{\mathrm{m}}+\Lambda, \quad-2 \dot{H}-3 H^{2}+\frac{1}{3 a^{2}} \delta^{i j} \Delta G_{i j}=\kappa^{2} P_{\mathrm{m}}-\Lambda,
$$


where $\Delta G_{00}$ and $\Delta G_{i j}$ are the modifications coming from the nonlocal terms:

$$
\begin{aligned}
\Delta G_{00} & =\left[3 H^{2}+3 H \partial_{t}\right]\left\{f\left(\square^{-1} R\right)+\square^{-1}\left[R \frac{d f}{d\left(\square^{-1} R\right)}\right]\right\} \\
& +\frac{1}{2} \partial_{t}\left(\square^{-1} R\right) \partial_{t}\left(\square^{-1}\left[R \frac{d f}{d\left(\square^{-1} R\right)}\right]\right), \\
\Delta G_{i j} & =a^{2} \delta_{i j}\left[\frac{1}{2} \partial_{t}\left(\square^{-1} R\right) \partial_{t}\left(\square^{-1}\left[R \frac{d f}{d\left(\square^{-1} R\right)}\right]\right)\right. \\
& -\left[2 \dot{H}+3 H^{2}+2 H \partial_{t}+\partial_{t}^{2}\right]\left\{f\left(\square^{-1} R\right)+\square^{-1}\left[R \frac{d f}{d\left(\square^{-1} R\right)}\right]\right\} .
\end{aligned}
$$

(b) Identifying now the scalar fields $\psi$ and $\xi$ with corresponding terms in the original action,

$$
\psi(t)=\square^{-1} R, \quad \xi(t)=\square^{-1}\left[R \frac{d f}{d\left(\square^{-1} R\right)}\right] .
$$

These fields $\psi(t)$ and $\xi(t)$ satisfy indeed the local equations.

(c) We conclude that these solutions are solutions of the initial nonlocal model as well, what can be checked immediately by direct substitution.

Some important comments are here in order.

1. The initial nonlocal model might be non-equivalent to its local formulation. Non-equivalence does not arise from a difference in the eqs., but from the initial (boundary) conditions.

2. Recasting the original form into the biscalar-tensor representation, one needs to invert the relationship $\psi=\square^{-1} R$, as $\square \psi=R$. For a given background, the solution for the latter equation is unique up to a harmonic function $\chi$ st $\square \chi=0$.

3. Compared to the original form, the scalar-tensor presentation seems to have introduced a new degree of freedom $\chi$. In fact, if we write $\psi \longrightarrow \psi+\chi$ the corresponding term is

$$
\xi(\square \psi-R) \longrightarrow \xi(\square(\psi+\chi)-R),
$$

after integration by parts, the change is $g^{\mu v} \partial_{\mu} \xi \partial_{\nu} \psi \longrightarrow g^{\mu v} \partial_{\mu} \xi \partial_{v}(\psi+\chi)$. It seems as if one had added an extra degree of freedom to the Lagrangian, absent in the original form.

4. However, imposing appropriate $\mathrm{BC}$, as $\chi=0$, to recover the original form, this would-be extra degree of freedom can be eliminated. The issue of the choice of correct BCs should be the only non-equivalence between original form and biscalar-tensor representation. Thus, e.g., Woodard et al. determine the inverse d'Alembertian operator using the retarded Green function: they fix a solution of Eq. $\square R=0$ by putting $\tilde{t}_{0}=0$ and $\eta_{0}=0$.

5. And, as a final but by no means less important comment note that, as we have stated above, the biscalar-tensor representation introduces two scalars, $\psi$ and $\xi$, therefore, working in this way it seems that one will encounter a ghost-like behavior. However, since the original 
nonlocal model does not introduce any new degree of freedom, the ghost-like behavior of the biscalar-tensor theory may not be physically relevant: associated terms can be cast as a boundary term of the nonlocal operators. At classical level, a necessary way to check this physical relevance is by considering the equivalence of the solutions coming from the original nonlocal formulation and from its biscalar-tensor form.

The action and equations of motion in the Einstein frame can be also constructed, after some work. And concerning the Jordan and Einstein frames, once a modified gravity theory is recast into its scalar-tensor presentation, it immediately follows that both the Jordan frame (where the matter sector minimally couples to gravity) and the Einstein one (where the Ricci is linear but matter couples to gravity non-minimally) are truly available.

And they are indeed related by a conformal transformation: $g_{\mu v}=\Omega^{2} g_{\mu v}^{(E)}$ where the metric in Jordan frame is $g_{\mu \nu}$, and in the Einstein frame, $g_{\mu \nu}^{(E)}$. However, one has to take seriously into account that:

1. The conformal transformation connecting both frames cannot be simply interpreted as a coordinate transformation of the theory. This is probably the reason for the long debate on which of the frames is 'the physical one' (the mathematical equivalence of the two frames being quite clear).

2. It is interesting to check is the precise behavior of corresponding solutions in the Einstein frame to see if and how much they differ from those of the Jordan frame. The transition between these frames is a useful tool for the construction of power-law solutions in the Einstein one.

3. Power-law solutions in the Einstein frame for the model without matter and for the model with matter, in different cases have been constructed indeed (see [ㅍ] ] $)$.

4. In the vacuum case, it had been previously found (Zhang and Sasaki), that some power-law solutions in the Jordan frame correspond to other power-law ones in the Einstein one. We do extend this correspondence for the model with a matter sector, and use the conformal transformation to obtain special solutions in Einstein's very difficult to get directly solving the system.

\section{Some conclusions and a short outlook}

Those were the main issues and results of the presentation (see also [2] $]$ ).

1. In GR, power-law solutions of type $H=n / t$ correspond to models with a perfect fluid whose EoS parameter $w_{\mathrm{m}} \equiv P_{\mathrm{m}} / \rho_{\mathrm{m}}$ is related to the power-index by $w_{\mathrm{m}}=-1+2 /(3 n)$.

2. Interesting would be to try to find similar power-law solutions in modified gravity theories, and to see how much they deviate from those for GR. We considered power-law solutions in a class of nonlocal gravity models stemming from the promising function $f\left(\square^{-1} R\right)=$ $f_{0} e^{\alpha \square^{-1} R}$ and including a perfect fluid with constant EoS parameter $w_{\mathrm{m}}$ 
3. Recasting the original nonlocal action into a local form, we obtained power-law solutions, with and without a cosmological constant, both in the Jordan and in the Einstein frames. We showed that power-law solutions got in Jordan frame satisfy the original nonlocal equations. In the Jordan frame we proved that all power-law solutions are found that way.

4. In Einstein's frame, power-law solutions were obtained either by solving the EoM, or by performing a conformal transformation of the solutions obtained in Jordan's frame. For this purpose, the correspondence was extended to include the matter sector. Using this very powerful and non-trivial tool, the solutions for $w_{\mathrm{m}}=1 / 3$ and $w_{\mathrm{m}}=1$ were obtained. These are very difficult to get by directly solving the system, what proves the usefulness of the method.

5. It had been previously shown by our group that not only models with exponential $f(\psi)$ can have power-law and de Sitter solutions, since a sum of exponentials is valid too. Another generalization of this analysis is to include several perfect fluid components with different constant values of $w_{\mathrm{m}}$, what we expect to do next.

6. From de Sitter solutions, it is interesting to check different possibilities for the Universe evolution, as those obtained from these nonlocal models, from the inflationary dS stage to the late power-law Universe. To check for deviations from the standard GR case, and its explicit distinction from other modified gravities, will be the subject of future study.

7. Theories with higher derivatives often suffer from a ghost problem, namely a wrong sign in the kinetic term, resulting in dangerous instabilities. A sound aspect of the conformal transformation technique between frames here developed is that one can obtain corresponding ghost-free conditions.

8. The biscalar-tensor representation introduces two extra scalars and it can lead to a ghost problem. The equivalence between the initial nonlocal theory and the local formulation has not been established yet. However, the ghost-like behavior of the biscalar-tensor theory may not be a physical problem, since the associated terms can presumably be cast as boundary terms of the nonlocal operators (the would-be ghost mode might just correspond to an inappropriate choice of $\mathrm{BC}$ ).

This important last question will be addressed in future work and also the analysis of the stability of the solutions here encountered, of the ghost-free conditions, and of the cosmological perturbations corresponding to these models.

Acknowledgments. The author thanks the Organizers of the Conference, in particular Prof. A.A. Bytsenko and Prof. A.E. Gonçalves, for the kind invitation and very nice hospitality they provided in Londrina. The last part of this presentation is based on joint work with E.O. Pozdeeva, S.Yu. Vernov, and Y.-1. Zhang, what the author gratefully acknowledges. This work was supported in part by MINECO (Spain), grant PR2011-0128 and project FIS2010-15640, by the CPAN Consolider Ingenio Project, and by AGAUR (Generalitat de Catalunya), contract 2009SGR-994. 


\section{References}

[1] H. Shapley and H.D. Curtis, "The scale of the universe", Bulletin of the National Research Council, Vol. 2, Part 3, National Research Council of the National Academy of Sciences, Washington, D.C., 1921; M. Bartusiak, “The Day We Found the Universe”, Random House Digital, 2010.

[2] E. Hubble, The New York Times, Nov. 23, 1924, p. 6.

[3] E.C. Pickering, "Periods of 25 Variable Stars in the Small Magellanic Cloud", Harvard College Observatory Circular 173, 2 (1912).

[4] A.A. Penzias and R.W. Wilson, Astrophys. J. Lett. 142, 419 Ü421 (1965).

[5] A.G. Riess, et al., Astrophys. J. 516, 1009 (1998).

[6] S. Perlmutter, et al., Astrophys. J. 517, 565 (1999).

[7] V. de Lapparent, M.J. Geller, J.P. Huchra, Astrophys. J. (Letters) 302, L1ÛL5 (1986).

[8] http://mwhite.berkeley.edu/BAO/SantaFe07.pdf

[9] R. Tolman, Beam Line, Fall 1997, Vol. 27, No. 3, p. 19.

[10] http://stuff.mit.edu/athena/course/8/8. 286/OldFiles/www/slides13/lec01-euf13-4up.ps.gz

[11] J.A. Frieman, C.T. Hill, A. Stebbins, and I. Waga, Phys. Rev. Lett. 75, 2077 (1995); K. Coble, S. Dodelson, and J.A. Frieman, Phys. Rev. D55, 1851 (1997); R. Caldwell, R. Dave, and P.J. Steinhardt, Phys. Rev. Lett. 80, 1582 (1998); B. Ratra and P.J.E. Peebles, Phys. Rev. D37, 3406 (1998); C. Wetterich, Nucl. Phys. B302, 668 (1998); D. Huterer and M. S. Turner, Phys. Rev. D60, 081301 (1999).

[12] S.M. Carroll, V. Duvvuri, M. Trodden, and M.S. Turner, Phys. Rev. D70, 043528 (2004); S Capozziello, Int. J. Mod. Phys. D11, 483 (2002).

[13] S. Park and S. Dodelson, Phys. Rev. D87, 024003 (2013).

[14] S. Deser and R. Woodard, Phys. Rev. Lett. 99, 111301 (2007).

[15] A.M. Polyakov, Phys. Lett. B103, 207 (1981).

[16] S. Capozziello, E. Elizalde, S. Nojiri, S.D. Odintsov, Phys. Lett. B671, 193 (2009); N. Koshelev, Grav. Cosmol. 15, 220 (2009).

[17] N. Tsamis, R. Woodard, Annals Phys. 267, 145 (1998); S. Nojiri, S.D. Odintsov, Phys. Lett. B659, 821 (2008).

[18] C. Deffayet, R. Woodard, JCAP 0908, 023 (2009).

[19] E. Elizalde, S. Nojiri, S. Odintsov, Phys. Rev. D70, 043539 (2004); W. Zhao and Y. Zhang, Phys. Rev. D73, 123509 (2006); H. S̆tefančić, Phys. Rev. D71, 124036 (2005).

[20] K. Bamba, S. Nojiri, S. Odintsov, M. Sasaki, Gen. Rel. Grav. 44, 441321 (2012).

[21] E. Elizalde, E.O. Pozdeeva, S.Yu. Vernov, Y.-1. Zhang, J. Cosm. Astrop. Phys JCAP07, 034 (2013). 